\title{
Original article \\ Study of Cucurbita extract effect on changes of AGEs, lipid and glycemic profile and CRP in type 1 diabetic rats \\ Sayahi $M^{1}$, Shirali $S^{2}$
}

\begin{abstract}
$\underline{\text { Abstract }}$
Background: Clinically, diabetes is an important risk factor or a range of diseases including nephropathy,retinopathy, angiopathy and deficiency and it is increasing in prevalence according to some estimates. (Cucurbita pepo L.) from the cucurbitaceae family has a low calorie but high nutritional and medical value. The aim of this study was to observe the effect of cucurbita extract on serum factors of STZ induced diabetic rats including glucose level AGEs, lipid profile (cholesterol, HDL, LDL, and TG) and CRP. Material and methods: Diabetes type1 was induced to Male albino- wistar rats by STZ at a dosage of $50 \mathrm{mg} \mathrm{kg}^{-1} .17$ rat were divided into three groups randomly including diabetic treated with extract, diabetic control and normal group. Diabetic group were force fed orally by cucurbita extract. The animals were anesthetized with ether at the end of 30 days and the blood was collected by syringe from their hearts for estimation of plasma glucose, TG, HDL, LDL, total cholesterol, CRP and AGEs Results: After measuring the serum factors and compares the result of the three groups to each other meaningful difference that shows the positive effect of cucurbita extract has been seen. Conclusion: According to the results we suggest that squash extract could have positive effect on regulating some of blood elements like glucose which are under the bad effect of diabetes type1 in rats. But more investigations are needed in this field to demonstrate the real benefits of cucurbita in curing diabetes and its related signs.
\end{abstract}

Keywords: Plant extract; squash; glucose; rat; cholesterol; triglyceride; diabetes

DOI: http://dx.doi.org/10.3329/bjms.v17i1.35286

Bangladesh Journal of Medical Science Vol. 17 No. 01 January'18. Page : 84-87

\section{Introduction:}

The global prevalence of diabetes mellitus is rapidly increasing as a result of population aging, urbanization and associated life style changes .the number of people with diabetes mellitus worldwide has more than doubled over the past three decades. The number of people globally with diabetes mellitus is projected to rise to 439 million by 2030 , which represents $7.7 \%$ of the total adult population of the world aged $20-79$ years ${ }^{1]}$. Diabetes mellitus is a chronic disorder of carbohydrate, lipid and protein metabolism manifested by elevated blood glucose level. This disease is caused by a defect in cellular uptake of glucose due to either reduced insulin secretion or cellular resistance to insulin ${ }^{2}$. There are two classes of diabetes mellitus known as type 1 diabetes mellitus ( T1DM) and type 2 diabetes mellitus ( T2DM ). T1DM is much less common with only $5-10 \%$ of all diabetes cases being type 1 . This type of diabetes usually presents it early in life though can occur at any age with some cases not being seen until the patient elderly. It is caused by damage to beta cells, which produce insulin, due to an auto- immune reaction. This damages causes greatly reduced or absent insulin production $^{3}$. Clinically, diabetes is an important risk factor or a range of diseases including nephropathy, retinopathy, angiopathy and deficiency and it is increasing in prevalence according to some estimates. The main reason is the beginning of a chain of chemical reaction $\mathrm{s}$ (Maillard reaction) after protein glycation, which

1. Miaad Sayahi, Student Research Committee, Ahvaz Jundishapur University of Medical Sciences, Ahvaz, Iran.

2. Saeed Shirali, Hyperlipidemia Research Center, Department of Laboratory Sciences, Faculty of Paramedicine, Ahvaz Jundishapur University of Medical Sciences, Ahvaz, Iran.

Correspondence to: Miaad Sayahi, Student Research Committee, Ahvaz Jundishapur University of Medical Sciences, Ahvaz, Iran. P.O.Box: 61375-15794, Ahvaz, Iran. E-mail: saeed.shirali@gmail.com, sayahi.m2014@ gmail.com 
results in the formation of the Schiff base, Amadori products, and, finally, the advanced glycation end products (AGEs). AGEs are nonfunctional protein aggregates that can change the structure and function of other proteins and can affect the signal transduction pathways ${ }^{4-6}$. Plants are often used in traditional medicine with over 200 species thought to be beneficial in the treatment of diabetes. The cucurbitaceae family, also referred to as cucurbits, are a group of fruit producing plants. They form a very large group with approximately 130 genera and 800 species and can be cultivated worldwide. Some of this species include squash, pumpkins, gourds and melons. Cucurbits are interesting because they have been exhibited extensive range of medicinal properties. It is believed that over 200 plants lower blood glucose level, including many common plants such as those belonging to the cucurbitaceae family ${ }^{3}$. This family classified as cucurbita pepo, cucurbita moschata, cucurbita maxima, and cucurbita mixta according to the texture and shape of their stems it contains chemicals , including proteins, fibers, tetra cyclicterpens, saponins, polysaccharides and minerals ${ }^{2}$. Cucurbitaceae is plant family generally considered to consist of melon, cucurbits and pumpkins ${ }^{7}$. The zucchini (cucurbita pepo L.), of the cucurbitaceae family, has a low calorie but high nutritional and medical value. These fruits can be found in many shapes, from spherical to elongated, and they vary in skin color from dark to light green, sometimes with fine white mottling or stripes ${ }^{8}$. Zucchini (cucurbita pepo L.), a small summer marrow or green squash, has a similar shape to a ridged cucumber'. The aim of thisstudy was toobserve the effect of cucurbita extract on serum factors of STZ induced diabetic rats including glucose level, lipid profile (cholesterol, HDL, LDL, TG) and AGEs in order to comparison the group treated with this extract withother groups which was as diabetic control and normal control .

\section{Material and method}

\section{Experimental animals:}

Male albino- wistar rats, weighing 180-200 g were obtained from the animal house of Ahvaz jundishapur University of medical sciences (Iran). Diabetes was induced by a single intraperitoneal injection of 0.1 $\mathrm{M}$ cold sodium citrate buffer ( $\mathrm{pH} 4.5)$ at a dosage of $50 \mathrm{mg} \mathrm{kg}^{-1}$ body weight. The animals were allowed to drink $5 \%$ glucose solution for a night in order to overcome the hypoglycemic effects induced by drug. The animals blood glucose values were measured by glucometer at $5^{\text {th }}$ day of STZ injection and they were considered as diabetic if the blood glucose was over $200 \mathrm{mg} \mathrm{dl}^{-1}$. The $6^{\text {th }}$ day after the STZ induction was considered as the $1^{\text {st }}$ day of treatment period. The rats were housed in in plastic cages with stainless metal cover in a room and were kept in the animal house during the period of treatment.

\section{Experimental set up:}

The experimental animals were divided into three groups. Untreated diabetic with 6 rats, normal controls with 6 rats and 5 diabetic rats treated with squash (cucurbita Pepo L.) extract and they were force fed orally by gavage with $500 \mathrm{ml} \mathrm{kg}^{-1}$ actually 3 $\mathrm{ml}$ per day according to rats weigh.

The animals were anesthetized with ether at the end of 30 days and the blood was collected by syringe from their hearts for estimation of plasma glucose, TG, HDL, LDL, total cholesterol and AGEs ${ }^{10}$.

\section{Preparation of extract:}

Fresh ripe fruits of squash (Cucurbita pepo L.) were obtained from local market. The plants were grown in Khuzestan, Iran. The average diameter of fruits was $7 \mathrm{~cm}$. after being leaned with water and antibacterial detergent. The whole fruit were processed in an electric domestic extractor, and the juice was collected. Generally $80 \mathrm{ml}$ of the extract was obtained from each $100 \mathrm{~g}$ of the starting crude materia ${ }^{11}$.

\section{Experimental design}

The albino rats were randomly assigned three groups (A-C) as follows:

Group A-was used as the normal control group consisted of 6 rats

Group B- diabetic rats treated with squash extract consisted of 5 rats

Group C- untreated diabetic rats consisted of 6 rats.

\section{Sampling method}

At the end of the study bleeding was done for blood factors measurement on the day 30. After anesthesia, bleeding was directly done from heart of rats, then blood samples were collected in tubes, 10 min after staying blood samples in laboratory environment, they were centrifuged and then serum harvested for determining the results ${ }^{12,13}$.

\section{Measurement}

The measurements were based on enzymatic colorimetric methods and were done by auto analyzer. HDL levels after fouling other lipoprotein's load in serum with magnesium chloride and phosphotungistic acid was determined using enzymatic colorimetric methods. AGE's measurement was performed by fluorimetry methods. Glucose measurement also was done by using enzymatic colorimeteric Methods. 
$\underline{\text { Statistical Analysis }}$

All the data are expressed as mean \pm SEM. Statistical comparisons were performed by one way analysis of variance (ANOVA). The results were considered statistically significant if the $p$ values were less than $0.05 \mathrm{in}$ most of the cases according to table1. The data were analyzed using SPSS vision $16.0^{12}$.

All the Results are set in a table1.

\begin{tabular}{|l|l|l|l|l|}
\hline $\begin{array}{l}\text { Groups } \\
\text { variable }\end{array}$ & \multicolumn{1}{|c|}{$\mathrm{A}$} & \multicolumn{1}{|c|}{$\mathrm{B}$} & $\mathrm{C}$ & $\mathrm{P}$ value \\
\hline glucose & $228.80 \pm 136.81$ & $103.17 \pm 19.13$ & $313.40 \pm 188.53$ & $\mathrm{P}=0.05 *$ \\
\hline triglycerides & $172.40 \pm 158.85$ & $93.16 \pm 23.18$ & $92.40 \pm 22.67$ & $\mathrm{P}=0.29$ \\
\hline $\begin{array}{l}\text { Total } \\
\text { cholesterol }\end{array}$ & $72.80 \pm 11.79$ & $73.83 \pm 9.86$ & $66.60 \pm 13.01$ & $\mathrm{P}=0.56$ \\
\hline HDL & $33.62 \pm 13.01$ & $38.76 \pm 4.46$ & $34.28 \pm 7.18$ & $\mathrm{P}=0.57$ \\
\hline LDL & $14.60 \pm 2.40$ & $13.16 \pm 0.98$ & $14.60 \pm 2.40$ & $\mathrm{P}=0.48$ \\
\hline $\begin{array}{l}\text { C-reactive } \\
\text { protein }\end{array}$ & $0.40 \pm 0.25$ & $0.38 \pm 0.14$ & $0.56 \pm 0.27$ & $\mathrm{P}=0.42$ \\
\hline AGEs & $0.15 \pm 0.31$ & $0.44 \pm 0.18$ & $0.35 \pm 0.26$ & $\mathrm{P}=0.25$ \\
\hline
\end{tabular}

Table1. $\mathrm{A}=$ control group, $\mathrm{B}=$ diabetic group treated with squash extract, $\mathrm{C}=$ untreated diabetic group

\section{Results:}

\section{Discussion:}

Recently using photochemicals in diabetes treatment are interested ${ }^{21-23}$. Till now, several pharmacological properties have been reported for different kinds of pumpkin (cucurbita pepo) which include antihyperlipidemic, antioxidant, hepatoprotective ${ }^{14}$ anti-carcinogenic, anti-microbial ${ }^{15,16}$ and antidiabetic properties ${ }^{10}$. This study was conducted to investigate the effect of squash (cucurbita pepo L.) extract on glucose and other blood parameters like lipid profile, CRP and AGE's levels on diabetic rats. the results showed that $\mathrm{p}$ value for glucose was less than 0.05 , so According to that we can say there is a significant difference in glucose levels among the normal control group (A) and the diabetic group(B) treated with squash extract and the untreated diabetic group (C) . So it can be concluded that squash has a positive hypoglycemic effect in a dosage of 500 $\mathrm{ml} \mathrm{kg}{ }^{-1}$ on rats. The antidiabetic and ameliorative effects of cucurbitaceae especially pumpkin have already been shown during researches on rats. In fact, results obtained in this research have been confirmed before in other studies. As Xia and Wang in 2006 showed, pumpkin methanolic extract reduces blood glucose levels and increase insulin levels in streptozotocin induced diabetic rats. According to them the significant antihyperglycemic effect may be due to the potentiating of plasma Insulin effect by increasing either the pancreatic secretion of insulin from the existing $\beta$-cells or its release from the bound Form, as evidenced by the significant increase in the level of insulin by C. ficifolia fruit extract in diabetic rats[10]. in other study Gourgue and Champ and their colleagues in 1992 showed that pectin, as an important component of plant cell walls, a water-soluble fiber is found in abundance in squash and The presence of pectin itself in the squash is considered as a hypoglycemic factor[17]. Based on the results of Kazemiand her colleagues research in 1389 which were performed on 28 rats it has been proved that the average size of pancreatic islets in the group treated with pumpkin compared to the diabetic group was increased. This suggests the reconstructive and regenerative effect of pumpkin powder on pancreatic. Following this action beta cells increase resulting more insulin production and secretion. so we can say that this way the adverse effects caused by a deficiency of insulin in diabetes partly goes away $^{18}$.

Studying results suggest that generally for other parameters the $p$ values were higher than 0.05 so we couldn't say there is a meaningful difference among the three groups. We didn't notice a significant decrease in other parameters after treatment for 30 days. But maybe in long term treatment there would be positive change for them, because as other studies showed cucurbita pepo species can effect lipid profile and even other blood factors.Fibers reduce plasma LDL level by inhibiting the absorbtion of cholesterol and bile acids and improving the activity of LDL receptors, so it could be concluded that pumpkin reduces the effect of lipids through its fibers. Fiber can also reduce triglyceride levels by suppressin lipogenesis in the liver ${ }^{19,20}$. The lipidreducing properties of cucurbita pepo plant are partly attributed to the pectin present on it. Pectin enhances the activity of lipoprotein lipase in fat tissue and heart, resulting in higher absorption of triglyceride rich lipoproteins (VLDL and chylomicron) in tissues other than liver to promote their breakdown and therefore reducing triglyceride levels Previous data suggest that diets rich in pectin facilitate excretion of bile acids which lead to their synthesis increase from cholesterol 
in the liver and ultimately reduction of blood cholesterol levels. Since LDL contains the highest level of cholesterol, LDL is likely to deplete following a reduction in cholesterol levels ${ }^{2}$.

\section{Conclusion}

According to the results we suggest that squash extract could have positive effect on regulating some of blood parameters especially glucose which is under the bad effect of diabetes type 1 in rats. But more investigations are needed in this field to demonstrate the real benefits of cucurbita in curing diabetes and its related signs.

\section{Acknowledgement:}

This study was supported by the Student Research Committee of Ahvaz Jundishapur University of Medical Sciences(No.93s35).

The authors declare that there are no conflicts of interest.

\section{References:}

1. Chen, L., D.J. Magliano, and P.Z. Zimmet, The worldwide epidemiology of type 2 diabetes mellitus[mdash]present and future perspectives. Nat Rev Endocrinol, 2012. 8(4): p. 228-236.

2. Sedigheh, A., et al., Hypoglycaemic and hypolipidemic effects of pumpkin (Cucurbita pepo L.) on alloxaninduced diabetic rats. African Journal of Pharmacy and Pharmacology, 2011. 5(23): p. 2620-2626.

3. Simpson, R. and G.A. Morris, The anti-diabetic potential of polysaccharides extracted from members of the cucurbit family: A review. Bioactive Carbohydrates and Dietary Fibre, 2014. 3(2): p. 106-114.

4. Shirali, S., S. Zahra Bathaie, and M. Nakhjavani, Effect of Crocin on the Insulin Resistance and Lipid Profile of Streptozotocin-Induced Diabetic Rats. Phytotherapy Research, 2013. 27(7): p. 1042-1047.

5. Shirali S, Babaali H, Babaali S. A Comparative Study on the Effects of Incretin and Metformin on Lipid Profile and Oxidative Stress in Diabetic Rats. International Journal of Pharmaceutical Research \& Allied Sciences, 2016, 5(2):227-233

6. Bathaie S. Z., Mokarizade N., and Shirali S., "An overview of the mechanisms of plant ingredients in the treatment of diabetes mellitus," Journal of Medicinal Plants, vol. 11, no. 44, pp. 1-24,2012

7. Adams, G.G., et al., The hypoglycaemic effect of pumpkins as anti-diabetic and functional medicines. Food Research International, 2011. 44(4): p. 862-867.

8. Iswaldi, I., et al., Profiling of phenolic and other polar compounds in zucchini (Cucurbita pepo L.) by reversephase high-performance liquid chromatography coupled to quadrupole time-of-flight mass spectrometry. Food Research International, 2013. 50(1): p. 77-84.

9. Neves, F.I.G., M.C. Vieira, and C.L.M. Silva, Inactivation kinetics of peroxidase in zucchini (Cucurbita pepo L.) by heat and UV-C radiation. Innovative Food Science \& Emerging Technologies, 2012. 13: p. 158-162.

10. Xia, T. and Q. Wang, Antihyperglycemic effect of Cucurbita ficifolia fruit extract in streptozotocin-induced diabetic rats. Fitoterapia, 2006. 77(7-8): p. 530-533.

11. Acosta-Patiño, J.L., et al., Hypoglycemic action of Cucurbita ficifolia on Type 2 diabetic patients with moderately high blood glucose levels. Journal of Ethnopharmacology, 2001. 77(1): p. 99-101.

12. Smith, Y., I. Adanlawo, and O. Oni, Hypoglycaemic Effect of Saponin from the Root of Garcinia kola (bitter kola) on alloxan-induced diabetic rats. Journal of Drug
Delivery and Therapeutics, 2012. 2(6).

13. Amirian, M., et al., Effect of prenatal stress (immobilization) on blood glucose levels and body weight. International Journal of Diabetes in Developing Countries, 2015. 35(1): p. 3-7.

14. Makni, M., et al., Hypolipidemic and hepatoprotective effects of flax and pumpkin seed mixture rich in $\omega-3$ and $\omega-6$ fatty acids in hypercholesterolemic rats. Food and Chemical Toxicology, 2008. 46(12): p. 3714-3720.

15. Pan, H., et al., Effect of pumpkin extracts on tumor growth inhibition in S180-bearing mice. Pract. Prev. Med, 2005. 12: p. 745-747.

16. Park, S.-C., et al., Pr-1, a novel antifungal protein from pumpkin rinds. Biotechnology letters, 2010. 32(1): p. 125-130.

17. Gourgue, C.M., et al., Dietary fiber from mango byproducts: Characterization and hypoglycemic effects determined by in vitro methods. Journal of Agricultural and Food Chemistry, 1992. 40(10): p. 1864-1868.

18. Kazemi, S., etal.,Preventive Effect of Pumpkin (Cucurbita Pepo L.) on Diabetic Index and Histopathology of Pancreas in Alloxan-Induced Diabetes in Rats. Journal of Isfahan Medical School, 2011. 28(117).

19. Takada, R., M. Saitoh, and T. Mori, Dietary y-Linolenic Acid-Enriched Oil Reduces Body Fat Content and Induces Liver Enzyme Activities Relating to Fatty Acid [3-Oxidation in Rats. J. Nutr, 1994. 124: p. 469-474.

20. Romero, A.L., et al., The seeds from Plantago ovata lower plasma lipids by altering hepatic and bile acid metabolism in guinea pigs. The Journal of nutrition, 2002. 132(6): p. 1194-1198.

21. PashazanousiM, RaeesiM, ShiraliS. Chemical composition of the essential oil, antibacterial and antioxidant activities, total phenolic and flavonoid evaluation of various extracts from leaves and fruit peels of Citrus limon. Asian Journal of Chemistry, 2014, 24 (10), 4331

22. Hosseini S, Gorjian M, Rasouli L, Shirali S. A Comparison between the Effect of Green Tea and Kombucha Prepared from Green Tea on the Weight of Diabetic Rats. BIOSCIENCES BIOTECHNOLOGY RESEARCH ASIA, 2015, 12, 141-146

23. Ebrahimi E, Shirali S, Talaei R. The Protective Effect of Marigold Hydroalcoholic Extract in STZ-Induced Diabetic Rats: Evaluation of Cardiac and Pancreatic Biomarkers in the Serum. Journal of Botany, Volume 2016(2016), Article ID 9803928, 6 pages 